Forum 2016 · 31:433

DOI 10.1007/s12312-016-0152-3

Online publiziert: 5. Oktober 2016

๑) Springer-Verlag Berlin Heidelberg 2016

KREBSVERBAND

$\because:$ BADEN-WÜRTTEMBERG E.V.

Krebsverband Baden-Württemberg e.V., Stuttgart

\title{
Trauer um Frau Prof. Else Heidemann
}

serung bei Früherkennung und Behandlung von Brustkrebs beigetragen", so die stellvertretende Vorsitzende des Krebsverbandes, Marion von Wartenberg. Ihr Markenzeichen war die besonders aufmerksame Zuwendung zu ihren Patienten, zu den Studierenden, ebenso wie zu ihren Mitarbeitern und Kollegen. Sie motivierte alle, ihr Bestes zu geben. Gelungen ist ihr dies in ihrer gewinnenden, sehr freundlichen und charmanten Art, die ihr half, genauso konsequent wie beharrlich ihre Ideen in die Tat umzusetzen, und ihre Partner zu überzeugen.

30 Jahre stand sie dem Onkologischen Schwerpunkt Stuttgart e. V. (OSP) als Vorsitzende vor. Ganz eng mit ihrem Namen sind hier die Brückenschwestern, sind die Palliativmedizin in Stuttgart, die Mammakarzinom-Nachsorgeleitstelle, die Tumordokumentation und das Benchmarkingprojekt, sind die www. krebsgemeinschaft.de und zahlreiche Leitlinien zur Diagnostik und Therapie von Krebserkrankungen verbunden.

Qualitätssichernde Organisation, interdisziplinäre Zusammenarbeit und klinische Krebsregistrierung waren dabei für sie kein Selbstzweck sondern wichtige Bestandteile der klinik- und fachübergreifenden Zusammenarbeit, ohne die eine solide Behandlung mit der ihr eigenen besonderen menschlichen Zuwendung nicht möglich gewesen wäre.

Professor Else Heidemann war seit 1999 Mitglied des Vorstandes des Krebsverbandes Baden-Württemberg, Mitglied des Landesbeirates Onkologie sowie der Ethikkommission der Universität Tübingen.

2015 erhielt sie den Ehrenpreis des Krebsverbandes Baden-Württemberg, verliehen an die Brückenpflege in BadenWürttemberg - 25 Jahre Modellprojekte zur Versorgung Schwerkranker und Sterbender - 20 Jahre Brückenpflege.
Ebenso erhielt sie 2005 den Anerkennungspreis des Forschungs- und Entwicklungspreises des Krebsverbandes BadenWürttemberg.

Der Anerkennungspreis Qualitätsförderpreis Gesundheit Baden-Württemberg wurde ihr 2004 verliehen.

Wir sind mit ihren Patienten und Mitarbeitern traurig und bestürzt über diesen Verlust, wir empfinden aber auch ihrer Lebensleistung gegenüber eine hohe Dankbarkeit.

Wir verlieren mit Frau Professor Else Heidemann eine warmherzige Ärztin, geschätzte Kollegin, beharrliche Kämpferin, langjährige Wegbegleiterin und vorbildliche Persönlichkeit.

Unser tiefes Mitgefühl gilt ihrer Familie. Wir werden sie vermissen.

\section{Prof. Walter Aulitzky}

Vorsitzender

\section{Marion von Wartenberg}

Stellv. Vorsitzende

\section{Hubert Seiter}

Geschäftsf. Vorstand

Prof. Uwe Martens

Sprecher der ATO

\section{Kontakt}

Birgit Wohland-Braun

Krebsverband Baden-Württemberg e.V.

Adalbert-Stifter Straße 105

70437 Stuttgart

Tel: 0711 848-10770

E-Mail:info@krebsverband-bw.de 